\title{
Relationship between motor recovery and independence after sensorimotor-complete cervical spinal cord injury
}

\author{
Kramer, John L K ; Lammertse, Daniel P ; Schubert, Martin ; Curt, Armin ; Steeves, John D
}

\begin{abstract}
BACKGROUND: For therapeutics directed to the injured spinal cord, a change in neurological impairment has been proposed as a relevant acute clinical study end point. However, changes in neurological function, even if statistically significant, may not be associated with a functional impact, such as a meaningful improvement in items within the self-care subscore of the Spinal Cord Independence Measure (SCIM). OBJECTIVE: The authors examined the functional significance associated with spontaneously recovering upper-extremity motor function after sensorimotor-complete cervical spinal cord injury (SCI). METHODS: Using the European Multi-center Study about Spinal Cord Injury (EMSCI) data set, a retrospective analysis was undertaken of individuals with cervical sensorimotor-complete SCI (initial motor level, C4-C7). Specifically, changes in upper-extremity motor score (UEMS), motor level, and SCIM (total and self-care subscore) were assessed between approximately 1 and 48 weeks after injury $(\mathrm{n}=74)$. RESULTS: The initial motor level did not significantly influence the total UEMS recovered or number of motor levels recovered. SCIM self-care subscore recovery was significantly greater for those individuals regaining 2 motor levels compared with those recovering only 1 or no motor levels. However, the recovery in the SCIM self-care subscore was not significantly different between individuals recovering only 1 motor level and those individuals who showed no motor-level improvement. CONCLUSIONS: A 2 motor-level improvement indicates a clinically meaningful change and might be considered a primary outcome in acute and subacute interventional trials enrolling individuals with cervical sensorimotor-complete SCI.
\end{abstract}

DOI: https://doi.org/10.1177/1545968312447306

Posted at the Zurich Open Repository and Archive, University of Zurich

ZORA URL: https://doi.org/10.5167/uzh-68403

Journal Article

Originally published at:

Kramer, John L K; Lammertse, Daniel P; Schubert, Martin; Curt, Armin; Steeves, John D (2012). Relationship between motor recovery and independence after sensorimotor-complete cervical spinal cord injury. Neurorehabilitation and neural repair, 26(9):1064-1071.

DOI: https://doi.org/10.1177/1545968312447306 


\section{Neurorehabilitation and Neural Repair \\ http://nnr.sagepub.com/}

Relationship Between Motor Recovery and Independence After Sensorimotor-Complete Cervical Spinal Cord Injury

John L. K. Kramer, Daniel P. Lammertse, Martin Schubert, Armin Curt and John D. Steeves Neurorehabil Neural Repair 2012 26: 1064 originally published online 30 May 2012

DOI: $10.1177 / 1545968312447306$

The online version of this article can be found at:

http://nnr.sagepub.com/content/26/9/1064

Published by:

(9)SAGE

http://www.sagepublications.com

On behalf of:

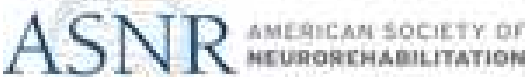

American Society of Neurorehabilitation

Additional services and information for Neurorehabilitation and Neural Repair can be found at:

Email Alerts: http://nnr.sagepub.com/cgi/alerts

Subscriptions: http://nnr.sagepub.com/subscriptions

Reprints: http://www.sagepub.com/journalsReprints.nav

Permissions: http://www.sagepub.com/journalsPermissions.nav

>> Version of Record - Oct 1, 2012

OnlineFirst Version of Record - May 30, 2012

What is This? 


\title{
Relationship Between Motor Recovery and Independence After Sensorimotor- Complete Cervical Spinal Cord Injury
}

Neurorehabilitation and Neural Repair

26(9) 1064-107I

(C) The Author(s) 2012

Reprints and permission: http://www. sagepub.com/journalsPermissions.nav DOI: I0.1 I77/I5459683|2447306

http://nnr.sagepub.com

\author{
John L. K. Kramer, PhD', Daniel P. Lammertse, MD², Martin Schubert, MD ${ }^{3,4}$, \\ Armin Curt, $M D^{3,4}$, and John D. Steeves, PhD'
}

\begin{abstract}
Background. For therapeutics directed to the injured spinal cord, a change in neurological impairment has been proposed as a relevant acute clinical study end point. However, changes in neurological function, even if statistically significant, may not be associated with a functional impact, such as a meaningful improvement in items within the self-care subscore of the Spinal Cord Independence Measure (SCIM). Objective. The authors examined the functional significance associated with spontaneously recovering upper-extremity motor function after sensorimotor-complete cervical spinal cord injury $(\mathrm{SCl})$. Methods. Using the European Multi-center Study about Spinal Cord Injury (EMSCl) data set, a retrospective analysis was undertaken of individuals with cervical sensorimotor-complete $\mathrm{SCl}$ (initial motor level, C4-C7). Specifically, changes in upper-extremity motor score (UEMS), motor level, and SCIM (total and self-care subscore) were assessed between approximately I and 48 weeks after injury $(n=74)$. Results. The initial motor level did not significantly influence the total UEMS recovered or number of motor levels recovered. SCIM self-care subscore recovery was significantly greater for those individuals regaining 2 motor levels compared with those recovering only I or no motor levels. However, the recovery in the SCIM self-care subscore was not significantly different between individuals recovering only I motor level and those individuals who showed no motor-level improvement. Conclusions. A 2 motor-level improvement indicates a clinically meaningful change and might be considered a primary outcome in acute and subacute interventional trials enrolling individuals with cervical sensorimotor-complete $\mathrm{SCl}$.
\end{abstract}

\section{Keywords}

spinal cord injury, Spinal Cord Independence Measure, recovery of function, upper extremity, activities of daily living, outcomes assessment

\section{Introduction}

Clinical researchers are faced with the ongoing challenge of choosing an appropriate clinical trial end point to determine the efficacy of a therapeutic intervention to ameliorate the neurological deficits after spinal cord injury (SCI) and/or improve functional outcomes. ${ }^{1-4}$ Currently, no gold standard exists for the treatment of SCI that would enable investigators to determine the relative benefits and merits for any novel neurologically targeted treatment. The selection of an appropriate clinical end point depends on a number of factors, including the trial phase, the intended clinical target, the desired patient population (eg, tetraplegia or paraplegia, complete or incomplete SCI), and the anticipated treatment effect, which could be subtle and confined to spinal cord segments adjacent to the site of SCI.

For therapeutics directed to the central nervous system (CNS), a change in the degree of neurological impairment, as defined by the International Standards for Neurological
Classification of Spinal Cord Injury (ISNCSCI), has been proposed as a relevant clinical end point. ${ }^{4,5}$ However, changes in neurological function, even if statistically significant between the experimental and control groups, may not be associated with a clear functional impact such as independent self-care (ie, a meaningful improvement in the activities of daily living [ADLs] or mobility). ${ }^{6}$ Functional

\footnotetext{
'International Collaboration On Repair Discoveries (ICORD),

University of British Columbia, and Vancouver Coastal Health,

Vancouver, BC, Canada

${ }^{2}$ Craig Hospital and University of Colorado at Denver, Englewood, CO, USA

${ }^{3}$ University Hospital Balgrist and University of Zurich, Zurich, Switzerland ${ }^{4}$ European Multicenter Study in Spinal Cord Injury (EMSCl)
}

\section{Corresponding Author:}

John D. Steeves, PhD, International Collaboration On Repair Discoveries (ICORD), University of British Columbia, and Vancouver Coastal Health, 818 West 10th Avenue, Vancouver, BC, V5Z IM9, Canada

Email: steeves@icord.org 
ADLs can be evaluated with global assessment protocols, such as the Spinal Cord Independence Measure (SCIM), which is a more specific assessment of SCI than the Functional Independence Measure. ${ }^{7}$ The use of a functional activity measure as the primary end point for a clinical trial, however, may not be satisfactory when the therapeutic targets CNS tissue regeneration. For example, SCIM outcomes are subject to the potential confounding influence of independent variables such as rehabilitation efforts. Thus, a challenge for clinical trials is to relate any measured neurological recovery to a clinically meaningful improvement in functional activities.

Recently, we examined the recovery of upper-extremity motor score (UEMS) and improvement in the ISNCSCIdefined motor level during the course of spontaneous neurological recovery after cervical sensorimotor-complete (ASIA Impairment Scale A, AIS-A) SCI. ${ }^{5}$ Motor level is defined as the most caudal spinal segment, as indexed by the key muscle group for that segment, having a muscle strength score of at least $3 / 5$ (full range contraction against gravity alone) while all the more rostral key muscles are normal $(5 / 5)$. Briefly, at the end of the first year, we observed a mean recovery of 5 motor points unilaterally within an upper extremity (maximum unilateral UEMS $=25$ ). In addition, up to $70 \%$ of the individuals with C5-C7 AIS-A SCI recovered at least 1 motor level (ie, caudal shift of motor level by 1 cervical segment), which has been previously reported. ${ }^{8}$ In contrast, only about $20 \%$ to $30 \%$ of individuals were reported to have recovered 2 or more motor levels unilaterally after 1 year of recovery. ${ }^{5}$ A lesser percentage of motor-level recovery was usually observed at intermediate time points.

In an earlier cross-sectional study, functional independence according to the SCIM was positively correlated with cervical motor level of SCI at 6 months after injury. ${ }^{9}$ The authors concluded that for each additional cervical motor level recovered, an individual is likely to recover significantly more functional independence. However, the detailed relationship between changes in motor score or motor level with SCIM during spontaneous recovery was not examined. Therefore, the primary aim of this study was to examine the functional significance of spontaneously recovering upper-extremity motor levels after sensorimotor-complete cervical SCI. We hypothesized that individuals spontaneously recovering 2 or more motor levels would regain significantly greater functional independence according to the SCIM than those recovering fewer motor levels, and thus, a recovery of 2 motor levels would represent an appropriate dichotomous primary trial end point to measure the efficacy of a future treatment.

\section{Methods}

\section{Data Set}

Individuals in the prospective European Multicenter Study about Spinal Cord Injury (EMSCI at www.emsci.org) that began in 2001 and currently includes 2317 participants with a traumatic SCI were included. The 18 European centers monitor neurological (ie, ISNCSCI) and functional (eg, SCIM) outcomes during the first year of standard care. The purpose of this ongoing collaboration is to evaluate the usual course of spontaneous neurological and functional recovery. Participants undergo an initial examination acutely ( $\sim 1$ week, but up to 15 days) after SCI, with followup assessments at $4,12,24$, and 48 weeks. Trained examiners perform the assessments. Other findings from this database have been published. ${ }^{5,9-14}$ The present study focused on the relationship between ISNCSCI motor outcomes $^{15}$ and SCIM ${ }^{16-18}$ during the year after onset. The most valid SCIM items, relating to upper-extremity motor recovery after cervical sensorimotor-complete (AIS-A) SCI, are found within the domain of self-care ${ }^{12}$ for personal grooming, feeding, bathing (upper and lower body), and dressing (upper and lower body). EMSCI initiated use of the SCIM with version II and subsequently adopted SCIMIII. SCIM-II and SCIM-III are very similar in terms of selfcare items. The SCIM version that was originally used for participants at the initial examination after SCI was also used throughout their follow-up assessments.

Changes in the SCIM total score, SCIM self-care subscore, total UEMS (bilateral aggregate motor score of the 5 upper-limb myotomes), and cervical motor level were checked between the initial examination and approximately 48 weeks after SCI. Motor-level changes (as defined above) were calculated for each side (right/left) separately.

\section{Inclusion and Exclusion Criteria}

Individuals who had traumatic cervical SCI that was sensorimotor complete (AIS-A) with an initial $\mathrm{C} 4$ to $\mathrm{C} 7$ motor level (right and left side) were included in this study. Because there are no key muscles to determine muscle strength in $\mathrm{C} 4$, the $\mathrm{C} 4$ motor level was determined according to ISNCSCI guidelines based on preservation of light touch and pinprick. Only those who had complete SCIM and neurological examinations at the initial examination and the final follow-up assessment at the final time point were included in the analysis. C8 and T1 levels of injury were excluded because of the small numbers of participants and because a 2 motor-level improvement within the upper extremities could not be determined.

\section{Statistics}

The mean change ( \pm standard deviation, $\mathrm{SD}$ ) scores between initial ( 1 to 15 days postinjury) and follow-up (approximately 48 weeks postinjury) examinations were used to describe motor and SCIM recovery. The 48-week time point was selected because this represents when the majority of spontaneous neurological recovery has been achieved. ${ }^{4,5,19}$ The frequency and proportions of individuals recovering 
motor levels was reported. Motor-level changes were examined bilaterally. That is, an individual was grouped according to the side (right or left) on which they recovered the greatest number of motor levels. Three motor-level groups were established: (1) no change or a deterioration of 1 or more motor levels, (2) 1 motor-level recovery, and (3) 2 or more motor levels of recovery. Individuals were grouped according to the greatest number of motor levels recovered. That is, if an individual recovered 1 motor level on the left side, but 2 motor levels on the right, they were grouped into the 2 motor-level recovery group. These groups were based on our previous analysis of the most common motor-level recovery patterns. ${ }^{5}$ The recovery of motor score (UEMS) and SCIM (total and self-care subscore) between initial and follow-up assessments was examined for significant differences based on the initial motor level (C4-C7, right and left side) using nonparametric statistics (Kruskal-Wallis $H$ ). Pairwise comparisons between initial cervical levels were Bonferroni corrected (Mann-Whitney $U$ ). The effect of initial cervical SCI level (C4-C7) on the frequency of motor-level changes was examined by a test of independent proportions. For the comparison of motor score and SCIM recovery based on the number of motor levels recovered, nonparametric statistics were also used (Kruskal-Wallis $H$ and Mann-Whitney $U$ for multiple pairwise comparisons). Because age has been shown to influence neurological and functional recovery, ${ }^{14,20}$ significant differences in age between initial motor level and the recovered motor-level groups were examined using nonparametric statistics (Kruskal-Wallis $H$ ). Alpha $(\alpha)$ was set at .05 , and all multiple pairwise comparisons for the motor-level groups were also Bonferroni corrected (adjusted $\alpha$ provided). All statistical procedures were performed in SPSS.

\section{Results \\ Participants}

A total of 157 individuals had sensorimotor-complete C4-C7 (both right and left sides) SCI at the initial assessment. Of these, 93 individuals had a complete motor evaluation at 48 weeks, and among these individuals, SCIM was also examined at both time points in 74 individuals (16 female patients). The cervical motor level of SCI was symmetrical on the right and left sides in $90 \%$ of cases at the initial examination. The average age was $39.8 \pm 18.5$ years.

\section{Initial Motor-Level and Motor Score/SCIM Recovery}

Age was not significantly different across the different initial cervical motor levels $\left(\chi^{2}=2.777 ; P=.427\right)$. Table 1 shows the average $( \pm \mathrm{SD})$ spontaneous recovery of the UEMS, motor level, and functional independence. Between 1
Table I. Spontaneous Recovery of UEMS, Number of Recovered Motor Levels, and SCIM After Cervical Sensorimotor-Complete Spinal Cord Injury

\begin{tabular}{|c|c|c|c|c|c|c|c|c|c|}
\hline \multirow{2}{*}{$\begin{array}{l}\text { Initial } \\
\text { Cervical } \\
\text { Motor Level }\end{array}$} & \multirow[b]{2}{*}{$\mathrm{n}$} & \multicolumn{2}{|c|}{ UEMS $\Delta^{\mathrm{a}}$} & \multicolumn{3}{|c|}{$\begin{array}{l}\text { Number of } \\
\text { Motor Levels } \\
\text { Recovered } \\
\text { (Percentage of } \\
\text { Valid n) }\end{array}$} & \multicolumn{2}{|c|}{$\begin{array}{c}\text { SCIM Total } \\
\Delta\end{array}$} & \multirow{2}{*}{$\begin{array}{c}\text { SCIM } \\
\text { Self-care } \\
\Delta \\
\text { Mean SD }\end{array}$} \\
\hline & & Mean & $S D$ & 0 & I & $\geq 2$ & Mean & SD & \\
\hline 4 & 27 & 9 & 11.3 & 48 & 29.6 & 22.2 & 18.4 & 5.2 & $2.7^{b} 5$ \\
\hline 5 & 29 & 11.7 & 7.5 & 20.7 & 51.7 & 27.6 & 27.6 & 17.0 & 6.45 \\
\hline 26 & II & 7.5 & 4.3 & 54.5 & 29.3 & 18.2 & 30.5 & 17.6 & 10.16 \\
\hline$C 7$ & 7 & 10.4 & 6.3 & 28.6 & 28.6 & 42.9 & 42.4 & 25.8 & 310.64 \\
\hline$=4-C 7$ & 74 & 10.7 & 8.6 & 36.5 & 37.8 & 25.7 & 26.1 & 18.1 & 6.06 \\
\hline
\end{tabular}

Abbreviations: UEMS, Upper-Extremity Motor Score; SCIM, Spinal Cord Independence Measure; SD, standard deviation.

${ }^{\mathrm{a}} \Delta$ is the change from initial to follow-up examinations.

${ }^{b} \mathrm{SCIM}$ self-care recovery significantly different from all other initial cervical levels of injury.

and 48 weeks, the initial cervical motor level did not significantly influence the total UEMS recovered $\left(\chi^{2}=2.777\right.$; $P=.427)$. At 48 weeks, the overall pattern and frequency of motor-level changes was the same for all initial cervical motor levels (C4-C7). Age was not significantly different between motor-level recovery groups $\left(\chi^{2}=3.074 ; P=.380\right)$ and did not influence outcomes.

The initial cervical motor level significantly influenced the recovery of total SCIM and SCIM self-care subscore $\left(\chi^{2}=12.023, P=.007\right.$, and $\left.\chi^{2}=23.333, P<.001\right)$. Individuals with an initial C4 motor-level injury recovered significantly fewer SCIM self-care points than those with injury to all other initial motor levels (adjusted $\alpha=.008$; all comparisons, $P \leq .001)$. After Bonferroni correction, none of the other pairwise comparisons between initial cervical motor levels and total SCIM recovery was significant (all comparisons, $P>.008$ ).

\section{Motor-Level and SCIM Recovery}

In approximately $80 \%$ of cases (see Table 2 ), changes in cervical motor levels were symmetrical between 1 and 48 weeks after C4-C7 sensorimotor-complete SCI (ie, motor level either deteriorated bilaterally, did not change on either the right or left side, or recovered the same number of segments bilaterally). As expected, UEMS recovery was significantly different depending on the number of motor levels recovered $\left(\chi^{2}=38.057, P<.001\right.$; Table 3 and Figure 1$)$. After correcting for multiple comparisons, UEMS recovery was significantly different for all pairwise comparisons $(P \leq .002)$.

SCIM total score and the self-care subscore were also significantly different depending on the number of motor 
Table 2. Right and Left Motor-Level Changes During Spontaneous Recovery

\begin{tabular}{llccc}
\hline Right $\Delta$ & \multicolumn{1}{c}{ Left $\Delta^{\mathrm{a}}$} & $\mathrm{n}$ & MLR Group & $\mathrm{n}$ \\
\hline Deterioration & Deterioration & $6^{\mathrm{b}}$ & Deterioration/no change & 27 \\
No change & No change & $21^{\mathrm{b}}$ & & \\
Deterioration & I MLR & I & I MLR & 28 \\
No change & I MLR & 4 & & \\
I MLR & No change & 3 & & \\
I MLR & I MLR & $20^{\mathrm{b}}$ & & \\
I MLR & 2 MLR & 4 & 2 MLR & 19 \\
2 MLR & I MLR & 3 & & \\
2 MLR & 2 MLR & $12^{\mathrm{b}}$ & & 74 \\
Total & & 74 & & \\
\hline
\end{tabular}

Abbreviation: MLR, motor-level recovery.

${ }^{\mathrm{a}} \Delta$ is the change from initial to follow-up examinations.

bymmetrical motor-level recovery.

Table 3. UEMS and SCIM Spontaneous Recovery After Cervical Sensorimotor-Complete Spinal Cord Injury as a Function of the Number of Motor Levels Recovered ${ }^{\mathrm{a}}$

\begin{tabular}{|c|c|c|c|c|c|c|c|}
\hline \multirow[b]{2}{*}{ Motor-Level Recovery } & \multirow[b]{2}{*}{$\mathrm{n}$} & \multicolumn{2}{|c|}{ UEMS $\Delta$} & \multicolumn{2}{|c|}{$\begin{array}{c}\text { SCIM Total } \\
\Delta\end{array}$} & \multicolumn{2}{|c|}{$\begin{array}{l}\text { SCIM Self- } \\
\text { care } \Delta\end{array}$} \\
\hline & & Mean & SD & Mean & SD & Mean & $\overline{S D}$ \\
\hline$\leq 0$ & 27 & 4.8 & 6.0 & 18.4 & 11.8 & 3.0 & 4.5 \\
\hline 1 & 28 & 11.3 & 5.3 & 25.59 & 16.4 & 5.7 & 5.7 \\
\hline$\geq 2$ & 19 & 18.1 & 9.9 & 37.6 & 22.2 & 10.6 & 5.9 \\
\hline 0 to $\geq 2$ & 74 & 10.7 & 8.6 & 26.1 & 18.1 & 6.0 & 6.1 \\
\hline
\end{tabular}

Abbreviations: UEMS, Upper-Extremity Motor Score; SCIM, Spinal Cord Independence Measure; SD, standard deviation.

${ }^{a} \Delta$ is the change from initial to follow-up examinations. Significant pairwise comparisons for SCIM self-care are shown in Figure I. All pairwise comparisons of UEMS recovery were significant.

levels recovered $\left(\chi^{2}=11.580, P=.003\right.$, and $\chi^{2}=18.538$, $P<.001$; Table 3 and Figure 1). However, neither SCIM total score nor the SCIM self-care subscore recovery was significantly different between those individuals whose motor level did not change or deteriorated and those individuals recovering only 1 motor level (adjusted $\alpha=.02$, $P=.162$, and $P=.06$, respectively). SCIM total score and SCIM self-care subscore were significantly greater for those individuals recovering 2 motor levels compared with those individuals who showed no motor-level improvement (ie, unchanged or deteriorating motor level; $P=.001$ and $P<.001$, respectively). SCIM self-care subscore recovery, but not total SCIM, was also significantly greater for those individuals regaining 2 motor levels compared with 1 motor-level recovery $(P=.005)$.

A small proportion of individuals with $\mathrm{C} 4-\mathrm{C} 7$ sensorimotor-complete SCI spontaneously recovered more than 2 motor levels $(n=5)$. It could be argued that combining these individuals with those who recovered exactly

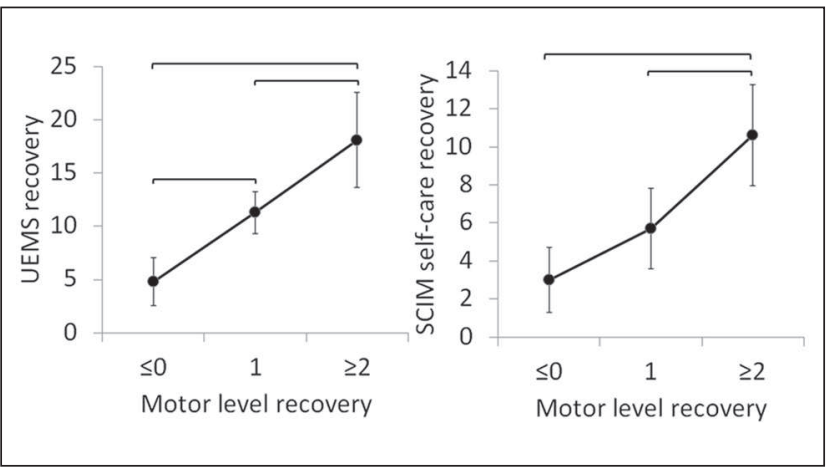

Figure I. UEMS and SCIM self-care score recovery after cervical (C4-C7) sensorimotor-complete (AIS-A) SCl between initial and follow-up examinations based on the number of motor levels recovered in that same period of time: error bars represent $95 \%$ confidence intervals. Significant pairwise comparisons are highlighted with horizontal bars $(\alpha<.05)$. There were no significant differences between the recovery of SCIM self-care subscore when 0 and I motor level was recovered (right panel), despite a significant increase in motor points (left panel). There was no significant difference between I and 2 motor-level recovery with regard to the total SCIM score. Abbreviations: UEMS, upperextremity motor score; SCIM, Spinal Cord Independence Measure; AIS, ASIA Impairment Scale; SCI, spinal cord injury.

2 motor levels may account for the statistical differences between SCIM improvements in the 1 and $\geq 2$ motor-level recovery groups (Figure 1). Because this would affect our interpretations, we undertook an analysis of SCIM recovery where individuals recovering greater than 2 motor levels were excluded from analysis. The statistical difference in the recovery of the SCIM self-care subscore between the 1 and 2 motor-level recovery groups remained significant $(P=$ .018 ), thereby substantiating the potential functional importance of regaining 2 motor levels and the possible use of this metric as a clinical end point in a trial.

\section{Discussion}

The primary objective of this study was to assess the clinical and functional relevance for the recovery of motor levels after sensorimotor-complete (AIS-A) cervical (C4-C7) SCI. To this end, the recovery of SCIM items (total score and self-care subscore) based on the number of cervical motor levels recovered $(0,1, \geq 2)$ between 1 ( 1 to 15 days) and 48 weeks after SCI (Figure 1) was examined. The findings from this analysis support the hypothesis that the recovery of 2 motor levels represents a clinically and functionally meaningful neurological change after cervical sensorimotor-complete SCI. Although changes in motor level were generally accompanied by the recovery of UEMS, improvements in functional independence, as measured by the SCIM self-care, were significantly greater for those individuals recovering 2 or more motor levels 
compared with those recovering only 1 motor level. Therefore, the recovery of 2 motor levels could be considered a meaningful primary outcome in an acute or subacute interventional trial involving individuals with cervical sensorimotor-complete SCI to determine efficacy of a therapeutic targeted to CNS tissue.

The purpose of our analysis was to focus on neurological outcomes because central neurological substrates are the target of many acute therapeutic interventions. In comparison to appropriate controls, changes in neurological outcomes (ie, impairment) as a result of an applied treatment will indicate biological activity of the therapeutic on the target tissue. In addition, neurological measures, such as motor-level improvement, are less likely to be influenced by as many uncontrolled (independent) variables as activity assessments, such as SCIM. Activity measures are more susceptible to the independent influences of rehabilitation and/or patient motivation. When using an activity measure as a clinical trial end point, the independent variables alone may account for any observed functional improvement by a patient living with SCI. Thus, the current challenge was to link a reasonable, but statistically valid, threshold change in neurological improvement with a corresponding and meaningful recovery of independent ADLs, such as those tracked by the SCIM self-care items.

\section{Clinical Implications for Acute SCI Trials: Primary Efficacy}

The intent here is not to undermine the importance of any neurological or functional improvement after SCI (including the recovery of even a single motor level) but to establish a meaningful and reasonable threshold of neurological improvement to measure a therapeutic treatment effect in a clinical trial. As shown in the results, the recovery of 2 motor levels was a requisite for a significant improvement in SCIM outcomes and was accompanied by significantly greater motor recovery compared with recovery of either 0 or 1 motor level.

In contrast, the recovery of 1 motor level just missed achieving a statistically significant change in SCIM outcomes compared with no motor-level change $(P=.06)$. The lack of statistical recovery according to SCIM could be influenced by a variety of factors, including a relatively small sample size $(n=28)$. Alternatively, the SCIM self-care subscore may not be sufficiently sensitive to track the subtle changes in outcomes associated with only recovering 1 motor level. Perhaps what is more important from a clinical trial perspective is that the spontaneous recovery of 1 motor level is a rather frequent event (approximately 64\% recover at least 1 motor level). Thus, as a clinical trial end point, setting a threshold at 1 motor level of improvement runs the risk of ceiling effects when trying to discern statistically significant differences between experimental and control groups.
Previous studies have examined the relationship between UEMS and motor-level recovery in patients with C5-C7 tetraplegia but considered changes on 1 side only (right side) or the right and left side separately. ${ }^{5,8,19}$ Thus, the true proportion of individuals recovering 2 motor levels on either side has likely been underrepresented in these studies. In the present study, if only the right-side motor-level changes were considered, $20 \%$ of individuals with C4-C7 SCI recovered 2 motor levels. However, if motor-level improvement on either side is considered, the recovery of 2 motor levels is approximately $25 \%$. Unlike individuals after a stroke or a peripheral unilateral upper-limb plexus lesion, individuals with tetraplegia may use whichever upper extremity shows sufficient motor recovery to accomplish ADLs. Thus, substantial functional arm/hand improvement on 1 side alone can have a significant impact on independent self-care.

In addition, the recovery of 2 motor levels may be equally important for individuals with an initial $\mathrm{C} 4$ motor level, where such recovery would facilitate the use of the wrist extensors (C6) and, thus, provide some voluntary hand function (eg, tenodesis grasp). This would facilitate improved independence for drinking, washing, shaving, and dressing the upper body, all of which would cumulate in greater recovery as tracked by the SCIM self-care subscore. ${ }^{3}$ On inspection at 48 weeks of only those individuals with an initial bilateral $\mathrm{C} 4$ motor level, the SCIM self-care score did not improve (ie, remained at 0 ) when the motor level failed to improve (ie, remained at C4), even if these patients achieved an increased UEMS (up to 7 motor points). In addition, if only 1 motor level was recovered from an initial C4 motor level, the average SCIM self-care recovery was limited to less than a 3-point change (ie, less than the mean recovery of SCIM self-care). Conversely, in the majority of patients with an initial $\mathrm{C} 4$ motor level, a 2 motor-level improvement by 48 weeks was accompanied by an average recovery of greater than 6 points in the SCIM self-care score or twice the expected mean recovery. Although this secondary analysis is limited by a small sample size, it provides additional support that the recovery of 2 motor levels is a functionally meaningful threshold, including for individuals with an initial C4 motor level of cervical sensorimotor-complete SCI.

\section{Compensation and Neurological Recovery}

The development of compensatory strategies to achieve motor tasks (eg, grooming) learned during the course of rehabilitation after sensorimotor-complete SCI has been identified as a prominent mechanism underlying the improvement of functional outcomes. ${ }^{11}$ Therefore, relying on functional activity instruments (eg, SCIM) to measure the efficacy of a neurological therapeutic (provided acutely or subacutely) is challenged by the extent of compensation 
that might be achieved by the individual during recovery as well as by a variety of independent variables outside the control of most clinical trials. These include, but are not limited to, the motivation and ability of the individual to learn new movement strategies in rehabilitation, ongoing participation in extensive inpatient/outpatient rehabilitation activities, and the use of assistive devices. Therefore, the use of a neurological clinical end point, such as the recovery of 2 motor levels (as defined by ISNCSCI), represents a more direct and reliable measure of a therapeutic directed to CNS tissue, with the added dimension of being correlated with the recovery of a meaningful function in terms of improved independence for activities of self-care.

The initial cervical motor level (C4-C7) did not influence the pattern of neurological outcomes (ie, the recovery of UEMS or the proportion of individuals recovering motor levels) but did have an influence on functional outcomes (ie, the statistically smaller increase in SCIM scores from an initial C4 level of SCI). This suggests that adopting the SCIM as the primary outcome instrument in an acute or subacute clinical trial would require different threshold values for different rostrocaudal levels of cervical sensorimotor-complete SCI. In addition, if therapeutic efficacy was based on a SCIM score responder threshold (ie, percentage of individuals recovering $\times$ number of SCIM points), careful consideration would have to be given to the distribution of individuals with initial $\mathrm{C} 4$ level across the treatment and control groups.

\section{Limitations}

There is no consensus on what constitutes a minimally clinically important difference (MCID) for neurological outcomes after cervical sensorimotor-complete SCI. Whether the recovery of 2 motor levels after sensorimotorcomplete cervical SCI is clinically, as well as statistically, significant remains to be determined. The objective of this study was not to determine MCID. However, the MCID for neurological recovery has been repeatedly suggested as being at least equal to the smallest real difference (SRD) ${ }^{21-23}$ :

$$
\mathrm{SRD}=1.96 \times \sqrt{2} \times(\mathrm{SD} \times \sqrt{[1-\text { Reliability coefficient }]}) .
$$

A change of 2 motor points has been previously reported as the SRD for UEMS. ${ }^{24}$ Based on a reliability coefficient of 0.95 , an estimate of the SRD at 48 weeks of those 74 individuals examined in this study is maximally 3 motor points. The SRD depends on the SD, which may differ slightly between motor levels of injury and is inflated if all motor levels are grouped.

Thus, our estimate of SRD is based on the SD of individuals with C6 motor-level injury at 48 weeks. This subgroup of individuals was selected because they demonstrated the largest SD with a meaningful sample size $(n=20$,
$\mathrm{SD} \pm 5.51$ ). Because a 10 motor point recovery of the UEMS is expected spontaneously on average, a change of 13 or greater in the UEMS would be the SRD and could be suggested in the context of a clinical trial as "clinically meaningful." Based on this value, 14 of 19 individuals (74\%) who recovered 2 motor levels also recovered at least 13 motor points. However, only 8 of 28 participants $(29 \%)$ recovering 1 motor level and only 1 of 27 participants (4\%) with no recovery of any motor level managed to regain 13 points within the upper extremities. Therefore, in about three quarters of those exhibiting a recovery of 2 motor levels, this is also accompanied by the number of motor points in the UEMS that equates to the SRD and could possibly be related to a MCID. Only with future clinical trial data will the issue of what is a MCID after cervical sensorimotorcomplete SCI be resolved.

Our findings are based only on those individuals who were available and volunteered for follow-up examinations of ISNCSCI and SCIM out to a chronic time point (approximately 48 weeks) when most individuals have been discharged from an inpatient rehabilitation center. Thus, there is potential selection bias because of participant attrition and nonrandomized drop-out effects. Furthermore, the low number of cervical sensorimotor-complete individuals available for analysis may also limit the generalizability of our findings, particularly with regard to individuals with initial C6 $(\mathrm{n}=11)$ and C7 $(\mathrm{n}=7)$ motor levels of SCI. However, our findings are in agreement with previous examinations of the EMSCI database as well as studies examining other large databases insofar as the overall neurological and functional recovery observed over the first year after sensorimotor-complete SCI is similar. ${ }^{8,19}$

Because there are no key muscles for $\mathrm{C} 4$, the $\mathrm{C} 4$ motor level is imputed based on the sensory level findings and a lack of C5 functional motor activity $(<3 / 5)$. This is a potential source of error because an individual diagnosed initially with a C4 motor level could actually have impaired C4 ventral root function on one or both sides. Similarly, an individual being diagnosed with an initial $\mathrm{C} 5$ motor level is based on a score of 3 or 4 in the elbow flexors (C5 myotome is the first testable myotome with ISNCSCI). Such an individual might not actually have completely normal motor function in all rostral cervical segments. These lead to limitations in the direct assessment of motor function at $\mathrm{C} 4$ and more rostral segments, which are inferred and reliant on the degree of preserved sensory function in the relevant rostral cervical segment and could result in an underestimation of motor recovery for this subset of patients. At present, there is no consensus within the SCI clinical research community on a standardized assessment for motor activity/strength at the $\mathrm{C} 4$ cord segment that aligns with the numeric rating scales used for C5-T1.

The present analysis was also limited to individuals with sensorimotor-complete cervical (C4-C7) SCI. The 
population with sensorimotor-complete SCI represents a high-priority population for developing effective treatment strategies and is often conceived as a starting point for "first in human" studies. ${ }^{25}$ Nevertheless, sensitive, accurate, and reliable trial outcome measures applicable to individuals with incomplete cervical SCI must also be developed and validated. Because differences in the extent of damage and possible mechanisms for spontaneous recovery are likely more variable after incomplete SCI, identifying appropriate outcome measures is likely to be even more challenging. It is not known whether neurological outcomes (eg, ISNCSCI) will be reliable. Perhaps novel functional outcome instruments need to be considered, including those focused more specifically on tracking changes in upper-limb function, such as the recently described Graded Redefined Assessment of Strength, Sensibility, and Prehension (or GRASSP). ${ }^{26,27}$

\section{Conclusions}

Acute and subacute clinical trials of treatments intended to improve neurological function in persons living with SCI should show evidence for statistical significance between the experimental and control groups and hopefully a clinically significant improvement in functional activities. The nature of the clinical end point should be pragmatically easy to measure, and the threshold for such an end point should be based on the natural history of recovery for the trial target population. We observed that the recovery of 2 motor levels is associated with a significantly greater recovery of functional independence (self-care) compared with no motor-level recovery or only 1 motor level of recovery.

It is true that a statistically significant improvement in neurological motor score or motor level (ie, structure and function) does not directly demonstrate the magnitude of a functionally meaningful benefit. However, relying solely on an activity measure, such as SCIM, does not directly assess whether a therapeutic induces a statistically significant change in biological activity within the targeted neural tissue. In addition, relying on an activity measure alone (eg, SCIM) introduces the risk of independent variables (eg, recovery as a result of rehabilitation) contributing to and altering the accurate interpretation of a benefit being realized from the therapeutic intervention. The present data suggest that this challenging dilemma may be best answered by assessing and comparing the recovery rates between the experimental and control groups for an improvement of 2 motor levels (unilaterally or bilaterally) after cervical sensorimotor-complete SCI. The use of this clinical end point could be a dichotomous response criterion (clinical end point threshold) for use in acute or subacute clinical trials of a therapeutic targeted to injured spinal cord tissue of people with cervical sensorimotorcomplete SCI.

\section{Declaration of Conflicting Interests}

The author(s) declared no potential conflicts of interest with respect to the research, authorship, and/or publication of this article.

\section{Funding}

The author(s) disclosed receipt of the following financial support for the research, authorship, and/or publication of this article: John Kramer was funded by the Canadian Institute of Health Research and the Michael Smith Foundation for Health Research. John Steeves is presently a Peter Wall Distinguished Scholar in Residence at UBC. The authors acknowledge Dr José Zariffa for his constructive suggestions and Renè Koller for his assistance with the European Multi-center Study about Spinal Cord Injury (EMSCI) database. The EMSCI is funded by the International Foundation for Paraplegia.

\section{References}

1. Alexander MS, Anderson KD, Biering-Sorensen F, et al. Outcome measures in spinal cord injury: recent assessments and recommendations for future directions. Spinal Cord. 2009;47:582-591.

2. Ditunno JF Jr, Burns AS, Marino RJ. Neurological and functional capacity outcome measures: essential to spinal cord injury clinical trials. J Rehabil Res Dev. 2005;42:35-41.

3. Consortium for Spinal Cord Medicine. Outcomes following traumatic spinal cord injury: clinical practice guidelines for healthcare professionals. J Spinal Cord Med. 2000;23:289-316.

4. Steeves JD, Lammertse D, Curt A, et al. Guidelines for the conduct of clinical trials for spinal cord injury (SCI) as developed by the ICCP panel: clinical trial outcome measures. Spinal Cord. 2007;45:206-221.

5. Steeves JD, Kramer JK, Fawcett JW, et al. Extent of spontaneous motor recovery after traumatic cervical sensorimotor complete spinal cord injury. Spinal Cord. 2011;49:257-265.

6. Walker MD. Acute spinal-cord injury. $N$ Engl J Med. 1991;324:1885-1887.

7. Anderson K, Aito S, Atkins M, et al. Functional recovery measures for spinal cord injury: an evidence-based review for clinical practice and research. J Spinal Cord Med. 2008;31:133-144.

8. Ditunno JF Jr, Cohen ME, Hauck WW, Jackson AB, Sipski ML. Recovery of upper-extremity strength in complete and incomplete tetraplegia: a multicenter study. Arch Phys Med Rehabil. 2000;81:389-393.

9. van Hedel HJ, Curt A. Fighting for each segment: estimating the clinical value of cervical and thoracic segments in SCI. J Neurotrauma. 2006;23:1621-1631.

10. Zariffa J, Kramer JL, Fawcett JW, et al. Characterization of neurological recovery following traumatic sensorimotor complete thoracic spinal cord injury. Spinal Cord. 2011;49:463-471.

11. van Hedel HJ, Dokladal P, Hotz-Boendermaker S; EM-SCi Study Group. Mismatch between investigator-determined 
and patient-reported independence after spinal cord injury: consequences for rehabilitation and trials. Neurorehabil Neural Repair. 2011;25:855-864.

12. Rudhe C, van Hedel HJ. Upper extremity function in persons with tetraplegia: relationships between strength, capacity, and the spinal cord independence measure. Neurorehabil Neural Repair. 2009;23:413-421.

13. Wirth B, van Hedel HJ, Kometer B, Dietz V, Curt A. Changes in activity after a complete spinal cord injury as measured by the Spinal Cord Independence Measure II (SCIM II). Neurorehabil Neural Repair. 2008;22:279-287.

14. Jakob W, Wirz M, van Hedel HJ, Dietz V. Difficulty of elderly SCI subjects to translate motor recovery - "body function"into daily living activities. J Neurotrauma. 2009;26:2037-2044.

15. Marino RJ, Barros T, Biering-Sorensen F, et al. International standards for neurological classification of spinal cord injury. J Spinal Cord Med. 2003;26(suppl 1):S50-S56.

16. Anderson KD, Acuff ME, Arp BG, et al. United States (US) multi-center study to assess the validity and reliability of the Spinal Cord Independence Measure (SCIM III). Spinal Cord. 2011;49:880-885.

17. Catz A, Itzkovich M, Agranov E, Ring H, Tamir A. SCIMspinal cord independence measure: a new disability scale for patients with spinal cord lesions. Spinal Cord. 1997;35:850-856.

18. Itzkovich M, Gelernter I, Biering-Sorensen F, et al. The Spinal Cord Independence Measure (SCIM) version III: reliability and validity in a multi-center international study. Disabil Rehabil. 2007;29:1926-1933.

19. Marino RJ, Burns S, Graves DE, Leiby BE, Kirshblum S, Lammertse DP. Upper- and lower-extremity motor recovery after traumatic cervical spinal cord injury: an update from the national spinal cord injury database. Arch Phys Med Rehabil. 2011;92:369-375

20. Furlan JC, Fehlings MG. The impact of age on mortality, impairment, and disability among adults with acute traumatic spinal cord injury. J Neurotrauma. 2009;26:1707-1717.

21. Beckerman H, Roebroeck ME, Lankhorst GJ, Becher JG, Bezemer PD, Verbeek AL. Smallest real difference, a link between reproducibility and responsiveness. Qual Life Res. 2001;10:571-578.

22. Copay AG, Subach BR, Glassman SD, Polly DW Jr, Schuler TC. Understanding the minimum clinically important difference: a review of concepts and methods. Spine J. 2007;7:541-546.

23. Schuck P, Zwingmann C. The "smallest real difference" as a measure of sensitivity to change: a critical analysis. Int $J$ Rehabil Res. 2003;26:85-91.

24. Marino RJ, Jones L, Kirshblum S, Tal J, Dasgupta A. Reliability and repeatability of the motor and sensory examination of the international standards for neurological classification of spinal cord injury. J Spinal Cord Med. 2008;31:166-170.

25. Wirth E III, Lebkowski JS, Lebacqz K. Response to Frederic Bretzner et al. "Target populations for first-in-human embryonic stem cell research in spinal cord injury." Cell Stem Cell. 2011;8:476-478.

26. Kalsi-Ryan S, Beaton D, Curt A, et al. The Graded Redefined Assessment of Strength Sensibility and Prehension (GRASSP): reliability and validity. $J$ Neurotrauma. 2012;29:905-914.

27. Zariffa J, Kapadia N, Kramer JL, et al. Feasibility and efficacy of upper limb robotic rehabilitation in a subacute cervical spinal cord injury population. Spinal Cord. 2012;50:220-226. 